\title{
Matrix metalloproteinases in serum of Emery-Dreifuss muscular dystrophy patients ${ }^{\star}$
}

\author{
Irena Niebroj-Dobosz ${ }^{1 凶}$, Agnieszka Madej-Pilarczyk ${ }^{1}$, Michał Marchel$^{2}$, \\ Beata Sokołowska ${ }^{3}$ and Irena Hausmanowa-Petrusewicz ${ }^{1}$
}

${ }^{1}$ Neuromuscular Unit, Institute of Clinical and Experimental Medicine, Polish Academy of Sciences, Warszawa, Poland; ${ }^{2}{ }^{\text {st }}$ Department of Cardiology, Warsaw Medical University, Warszawa, Poland; Department of Respiratory Research, Institute of Clinical and Experimental Medicine, Polish Academy of Sciences, Warszawa, Poland

Received: 10 February, 2009; 08 September, 2009; accepted: 23 November, 2009

available on-line: 08 December, 2009

\begin{abstract}
In the pathogenesis of dilated cardiomyopathy (DCM) in Emery-Dreifuss muscular dystrophy (EDMD) matrix metalloproteinases (MMPs) are supposed to be involved and may have diagnostic/prognostic value. Serum levels of MT1-MMP, MMP-2 and MMP-9 were quantified by ELISA and zymography in 22 EDMD patients and 15 age-matched controls. In the autosomal-dominant EDMD MMP-2 and MT1-MMP were increased in all cases, and MMP-9 was increased in two of the eight examined patients. In the X-linked EDMD MMP-2 expression was increased in all the cases, MMP-9 level was elevated in 3 of the 14 cases, and MT1-MMP was decreased in eight of these patients. There was no evident correlation between the MMPs level and the different cardiac parameters including left-ventricular end-diastolic diameter, left atrial diameter and left ventricular ejection fraction in either form of EDMD. The presented results indicate that a changed level of matrix metalloproteinases, especially that of MMP-2 in serum, may be of value for detection of cardiac involvement in EDMD patients, especially in those patients with no evident subjective cardiac symptoms. Further follow-up studies of MMPs are needed to check if their determination is of value for monitoring of the progression of atrial/ventricular dilatation. MMPs determinations may also be useful for monitoring DCM treatment by synthetic MMPs inhibitors.
\end{abstract}

Keywords: Emery-Dreifuss muscular dystrophy, dilated cardiomyopathy, matrix metalloproteinases

\section{INTRODUCTION}

The deficit of lamins $\mathrm{A} / \mathrm{C}$ or emerin in the skeletal and heart muscle contribute to a rare, autosomal-dominant or X-linked Emery-Dreifuss muscular dystrophy (EDMD). Although the defect is generalized, selectively affected are skeletal muscles, heart and joints. Among the dominant clinical symptoms are skeletal muscle atrophy, joint contractures and dilated cardiomyopathy, the latter being at the onset either clinically silent, or preceded by arrhythmias and conduction block. The pathogenesis of dilated cardiomyopathy (DCM) in EDMD is not recognized yet. It may be also evoked by changes in myocardial extracellular matrix (ECM) which is responsible for ventricular stability and aligement of cardiomyocytes (Brilla et al., 1995). Induction of matrix metalloproteinases (MMPs) in dilated cardiomyopathy is already described (Tyagi et al., 1996; Thomas et al., 1998; Spinale et al., 1999; Schwartzkopff et al., 2002; Ohtsuka et al., 2003; 2007). It is suggested that determination of MMPs may serve as valuable markers

\footnotetext{
${ }^{\square}$ Corresponding author: Institute of Clinical and Experimental Medicine, A. Pawińskiego 5, 02-106 Warszawa, Poland; tel./fax: (48) 22608 631; e-mail: dobosz@cmdik.pan.pl.

^ The study was presented at the 20th Neurological Congress in Wrocław, Poland, September 2008.

Abbreviations: AD, autosomal dominant; AF, atrial fibrillation; AVB, atrio-ventricular block; AFL, atrial flutter; DCM, dilated cardiomyopathy; ECM, extracellular matrix; EDMD, Emery-Dreifuss muscular dystrophy; EF, ejection fraction; HTX, heart transplantation; ICD, implanted cardioverter/defibrilator; LAD, left atrium diameter; LVDD, left ventricular end-diastolic diameter; MMPs, matrix metalloproteinases; PAF, paroxysmal atrial fibrillation; RBBB, right bundle branch block; SAB, sinoatrial block; SSS, sick sinus syndrome; SVT, superventricular tachycardia; SVEB, supraventricular ectopic beats; $\mathrm{VF}$, ventricular fibrillation; VPB, ventricular premature beats; VT, ventricular tachycardia; X-EDMD, X-linked EDMD.
} 
for progression of left ventricle dilatation. The participation of the matrix metalloproteinases system in DCM in course of EDMD has not been studied yet.

The aim of this study was to analyze if determinations of the levels of circulating MMPs may be useful in detection of cardiac involvement in EDMD. The identification of cardiac status in EDMD is of special interest because of considerable mortality and risk of sudden death in patients and carriers.

\section{PATIENTS AND METHODS}

Twenty-two patients (18 men, 4 women) with Emery-Dreifuss muscular dystrophy (EDMD) eight autosomal-dominant (AD-EDMD), $14-$ X-linked (X-EDMD), aged 30 \pm 11 (14-54 years) were enrolled in this study. AD-EDMD patients had a gene mutation in the LMNA, X-EDMD in the STA gene. In the course of the disease a generalized cardiomyopathy was diagnosed in all cases. The moment of appearance of cardiomyopathy is, however, very difficult to define in both EDMD forms. No subjective cardiac symptoms, even with evident bradycardia, are often present. In some patients cardiac involvement was already detected at the time of neurological diagnosis of EDMD.

In the AD-EDMD left ventricle dysfunction was present. There were disturbances of conductivity, or contractility (Table 1a). The progress of cardiac symptoms in four of the eight patients was severe, in four it was mild or moderate. Pacemaker was implanted in three patients, cardioverter/defibrilator was implanted in one patient, in one case heart transplantation was performed, one patient (KT) suddenly died. DCM developed earlier in the AD-EDMD than in the X-EDMD group.

In ten of X-EDMD patients (Table $1 b$ ) the cardiac involvement was moderate, in three patients it was mild, in one case severe. Among the cardiac parameters at the beginning of the disease conductance and atrial involvement predominated. DCM developed later as compared to the AD-EDMD group. In 11 of the 14 patients pacemaker was implanted.

The control group consisted of 15 agematched normal subjects with no history of cardiac symptoms.

Measurements of MMPs level in serum. MMP-2 was determined by a sandwich enzyme im- munoassay kit employing two mouse monoclonal antibodies (Calbiochem, USA). In the MMP-9 determinations a sandwich enzyme immunoassay procedure employing a mouse monoclonal antibody and a sheep polyclonal antibody was used (Calbiochem, USA). MT1-MMP was determined by a two-step sandwich immunoassay (Chemicon International, USA) with enzyme-labeled antibodies against different antigenic forms (active and its precursor). The absorbance was assessed using a Sigma Diagnostics EIA Microwell Reader II. For zymography serum samples were diluted 1:50 with phosphate-buffered saline. The zymogram of MMP-2 and MMP-9 was resolved on Bio-Rad 10\% precast gels with gelatin according to Azeh et al. (1998). The gels were stained with Coomassie Brilliant Blue R-250 and were further processed in GelDoc 1000/2000 system (BioRad), Multi-Analyst PC version 1.2 and Mitsubishi Video Printer P91.

Statistical analysis. The results were expressed as mean \pm standard deviation. For analysis of variance (ANOVA) on ranks the Kruskal-Wallis test was applied. Mann-Whitney test was used to compare both groups. The relations between variables were studied using Spearman's correlation coefficients. Data were analyzed by StatSoft statistical software package version 5 .

\section{RESULTS AND DISCUSSION}

In serum of the AD-EDMD patients the level of MMP-2 and MT1-MMP was significantly increased in all of the cases, an increase of MMP-9 (over 2 S.D. of the normal mean) was present only in two of the eight examined patients (Table 1a). In X-EDMD the values of MMP-2 were also increased in all of the patients, the increase of the MMP-9 level was on the border of significance (a significant increase appeared in three of these cases), the MT1-MMP level was decreased (in eight of the 14 cases) (Table 1b). The difference in MT1-MMP between AD-EDMD and X-EDMD was significant. Zymograms indicated increased activity of MMP-2 in all cases of EDMD, while an increased activity of MMP-9 appeared only in some of them (Fig. 1). No difference in the circulating matrix metalloproteinases was found between male and female patients. There was no evident correlation between the levels of particular MMPs nor

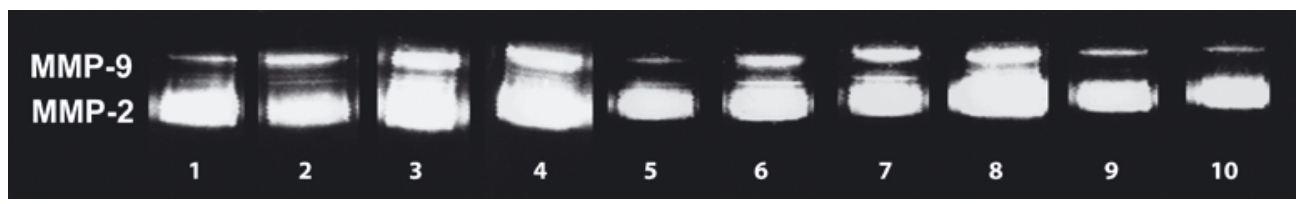

Figure 1. Representative gelatin zymogram of MMP-2 and MMP-9 in serum of EDMD patients.

AD-EDMD patients (lanes 1-4: GK, DS, NR, KT); X-EDMD patients (lanes 5-8: AD, KC, MK, TR); normal controls (lanes 9-10). 


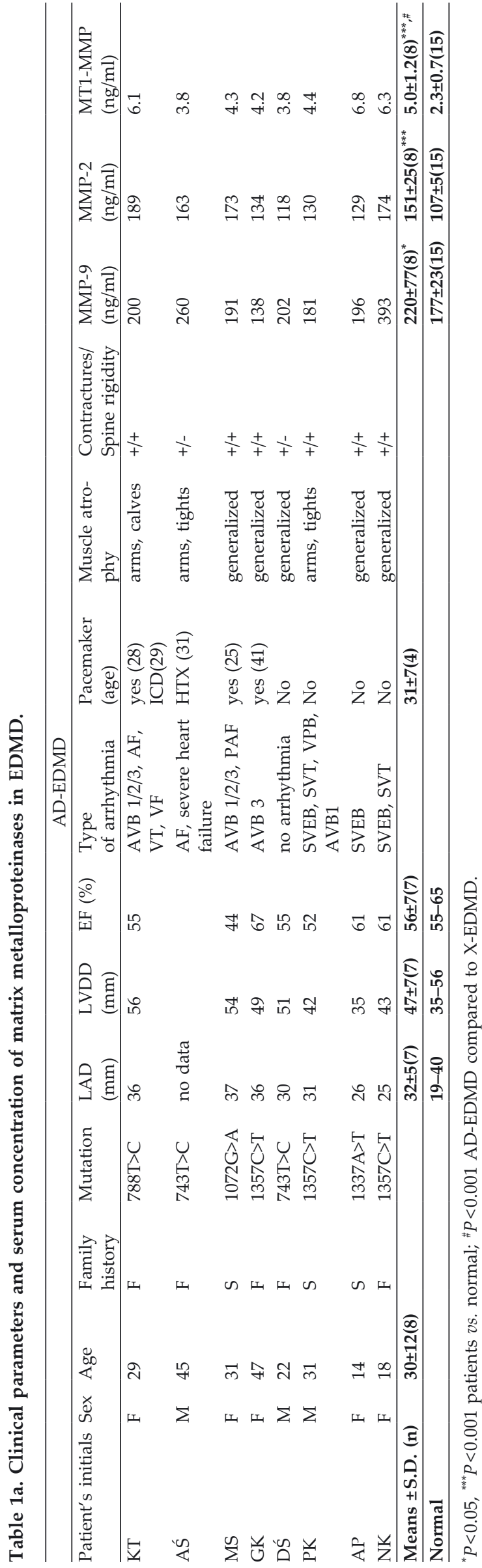

between the MMPs and different cardiac or other clinical parameters.

Matrix metalloproteinases are a family of over 20 zinc-dependent enzymes responsible for degradation of components of the extracellular matrix (ECM), which maintains myocardial geometry during cardiac action and cardiac dysfunction (Goldsmith \& Borg, 2002). Individual enzymes differ in substrate specificity and primary structure. Only some of them have been investigated to date (MMP2, MMP-3, MMP-9, MMP-1). MMPs are detected in normal and pathologically changed myocardium (Spinale, 2002). The activity of MMPs is regulated at the transcriptional, translational, and post-translational level, by endogenous extracellular signals (cytokines, hormones, a variety of peptide growth factors) (MacNaul et al., 1990; Ries \& Woessner, 1991; Ries \& Petrides, 1995) and specific inhibitors and regulators (TIMPs) (Weber \& Brilla, 1991; Woessner, 1991; Tyagi et al., 1995; Kähäri \& Saarialho, 1999). The activity of MMP-2, MMP-7, and TIMP-1-4 increases, that of MMP-9 decreases with age (Bonnema et al., 2007). The MMPs level is also under hormonal control. Female sex steroids inhibit MMPs (Singer et al., 2000). A link between noradrenaline and MMP-2 is also recognized (Banfi et al., 2005).

A number of cardiovascular diseases (dilated cardiomyopathy, hyperthropic cardiomyopathy, heart infarct, congestive heart failure, arterial hypertension) are associated with increased synthesis and degradation of extracellular matrix and disturbed maturation and incorporation of the matrix components in the myocardium.

Cardiomyopathy is accompanied by increased deposition of collagen. Little is, however, known about the temporal relationship between collagen gene transcription, the occurrence of cardiac fibrosis, and digestion of collagen by MMPs. It is not solved yet if the activation of the collagenolytic system is of primary or secondary importance in the progression of left ventricle dilation. Nevertheless, degradation of the fibrillar collagen matrix by collagenases is suggested to contribute to ventricular dilation (Spinale, 2002) and to coincide with the remodeling of the left ventricle and the onset of dilatation.

Any imbalance between the MMPs and TIMP systems disturbs the myocardial architecture, as both MMPs and their tissue inhibitors (TIMPs) maintain the architecture of the extracellular matrix and contribute to left ventricle remodeling and the subsequent deterioration of left ventricle performance (Tyagi et al., 1995; Thomas et al., 1998; Yokoseki et al., 2000).

In idiopathic dilated cardiomyopathy there is an association between collagen degradation and increased expression of MMPs (Reddy et al., 2004). There are, however, controversies on the presence 


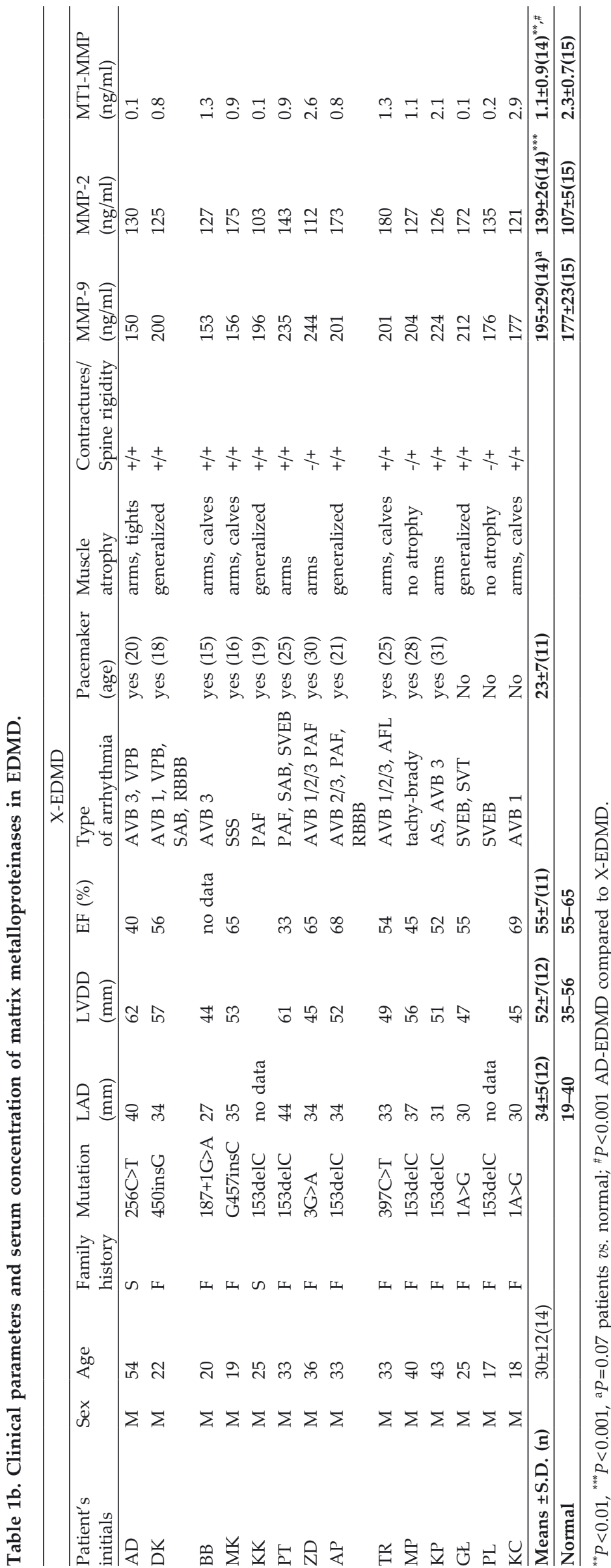

of alterations of particular MMPs and TIMPs (Tyagi et al., 1996; Li et al. 1998; Rouet-Benzineb et al. 1999; Spinale et al., 1999; 2000; Spinale, 2002; Thomas et al., 1998). In mild to moderate DCM the myocardial activity of MMP-2, MMP-3 and MMP-9 is elevated, that of MMP-1 decreased (Spinale et al., 1999). In the end-stage of this disease the left ventricle MMP-1 decreases, that of MMP3 and MMP-9 increases, the level of MMP-2 remains unchanged and TIMP1 is up-regulated (Thomas et al., 1998). An increase of MMP-2 and MMP-9, a decrease of TIMP-1 and TIMP-2 (Rouet-Benzineb et al., 1999), or increase in MMP-1 and down-regulation of TIMP1 (Tyagi et al., 1996), down regulation of TIMPs and up regulation of MMP-9 have been also reported ( $\mathrm{Li}$ et al., 1998). Selectively decreased in the myocardium is TIMP-3 (Fedak et al., 2004).

Although results of studies of particular MMPs conducted on dilated, ischaemic and valvular cardiomyopathies are divergent, they show that down/upregulation of collagenolytic activity conducted by MMPs is present (Tyagi et al., 1996; Spinale et al., 1999) and leads to ECM interruption and its disorganization (Beltrami et al., 1995; Pauschinger et al., 1999; Li et al., 2000). Overexpression of MT1-MMP and TIMP-1 mRNA in DCM also indicates increased collagenolytic activity (Picard et al., 2006). Besides their action on collagen, MMPs are also involved in disorganization of the contractile apparatus in DCM hearts by cleaving myosin heavy chain (RouetBenzineb et al., 1999).

Changes in the level of collagen degradation markers are reflected in serum of patients with different cardiovascular diseases. It is higher in idiopathic, as compared to ischemic DCM (Tziakas et al., 2005). An association between serum MMP-9 level and clinical cardiac symptoms with a risk of cardiovascular death has been also indicated (Blankenberg et al., 2003). Serious cardiac events also correlate with an increased MMP-3 level (Ohtsuka et al., 2007). In patients with congestive heart failure an increase of circulating MMP-2 (Yamazaki et al., 2004), or MMP-2, MMP-9 and TIMP-1 is observed (George et al., 2005), which 
correlates with the severity of the disease (Yamazaki et al., 2004). It is also indicated that MMP-1 and TIMP-1 levels in serum of DCM patients may serve as markers of progression of the left ventricle dilatation (Schwartzkopff et al., 2002). The progressive activation of MMPs in the course of DCM may be evoked by increased levels of circulating specific cytokines (Marriott et al., 1996; Munger et al., 1996).

In EDMD cardiac risk and the expression of cardiac involvement is highly variable. At the beginning of this disease no clinical cardiac symptoms are usually present. In the course of the disease EDMD patients and carriers often need a permanent cardiac pacemaker, in some cases sudden death occurs (Rowland et al., 1979; Miller et al., 1985; Galassi et al., 1986; Pinelli et al., 1987; Fishbein et al., 1993; Buckley et al., 1999). Progressive atrial fibrillation/flutter, atrial paralysis, heart block with slow junctional rhythm, and later on ventricular dilatation and ventricular failure appear. The myocardium is progressively replaced by fibrous and adipose tissues (Yoshioka et al., 1989). Early onset of severe myocardial fibrosis before clinical and echocardiographic signs and cardiac dysfunction is already manifested in a family with a deletion of the $5^{\prime}$ end of the LMNA gene (van Tintelen et al., 2007). An analysis of the MMPs/TIMPs system in EDMD patients, in the myocardium or serum has not been conducted until now.

Here we evaluated the potential significance of circulating MMPs levels in patients with EDMD. Serum level of MMP-2 was higher in all patients of both groups of EDMD. The difference in MT1-MMP expression between AD-EDMD and X-EDMD may be connected with the fact that a more severe cardiac involvement is present in the AD-EDMD form. MMP-9 level appears to be less informative, as its increase was only present in a small number of patients.

Further examination of MMPs, particularly of MMP-2, in EDMD patients, their relatives and carriers, especially in follow up studies, is needed. MMPs determination may be of value in prediction of the susceptibility to the dilated cardiomyopathy, especially important in cases with no preceding clinical cardiac symptoms. The MMPs determination may be also conducted in monitoring DCM treatment by means of synthetic MMPs inhibitors, which could prevent left ventricular dilatation and delay the impairment of left ventricular systolic function. The introduction, however, of synthetic inhibitors to the strategy of DCM treatment has to be preceded by detailed studies of the whole MMPs family, especially as the sensitivity of the individual enzymes to the inhibitors greatly differs.

\section{Acknowledgement}

This work was supported by Grant No. 2P05B 10629 of the State Committee for Scientific Research to I.H.-P.

The study was approved by the Ethics Committee for Human Research at the Warsaw Medical University. There were no conflicts of interest regarding the study presented in this paper.

\section{REFERENCES}

Azeh L, Mader M, Smirnov A, Beuche W, Nau R, Weber F (1998) Experimental pneumococcal meningitis in rabbits: the increase of matrix metalloproteinase- 9 in cerebrospinal fluid correlates with leucocyte invasion. Neurosci Lett 256: 127-130.

Banfi C, Cavalca V, Veglia F, Brioschi M, Barcella S, Mussoni L, Boccotti L, Tremoli F, Biglioli P, Agostoni P (2005) Neurohormonal activation is associated with increased levels of plasma matrix metalloproteinase- 2 in human heart failure. Eur Heart J 26: 481-488.

Beltrami CA, Finato N, Rocco M, Feruglio GA, Puricelli C, Cigola E, Sonnenblick ED, Olivetti G, Anversa P (1995) The cellular basis of dilated cardiomyopathy in humans. J Mol Cell Cardiol 27: 291-305.

Blankenberg S, Rupprecht HJ, Poirier O, Bickel M, Smieja M, Hafner G, Meyer J, Cambien F, Tiret L, AtheroGene Investigators (2003) Plasma concentrations and genetic variation of matrix metalloproteinase 9 and prognosis of patients with cardiovascular disease. Circulation 107: 1579-1585.

Bonnema DD, Webb CS, Pennington WR, Stroud RE, Leonardi AE, Clark LL, Mc Clure CD, Finklea L, Spinale FG, Zile MR (2007) Effects of age on plasma matrix metalloproteinase (MMPs) and tissue inhibitor of metalloproteinases (TIMPs). J Card Fail 13: 530-540.

Brilla CG, Maisch B, Zhou G, Weber KT (1995) Hormonal regulation of cardiac fibroblast function. Eur Heart $J 16$ (Suppl C): 45-50.

Buckley AE, Dean J, Mahy IR (1999) Cardiac involvement in Emery-Dreifuss muscular dystrophy: a case series. Heart 82: 105-108.

Fedak PW, Smookler DS, Kassiri Z, Ohno N, Leco KJ, Verma S, Mickle DA, Watson KL, Hojilla CA, Cruz W, Weisel RD, Li RK, Khokha R (2004) TIMP-3 deficiency leads to dilated cardiomyopathy. Circulation 110: 24012409.

Fishbein MC, Siegel RJ, Thompson ChE, Hopkins LC (1993) Sudden death of a carrier of X-linked Emery-Dreifuss muscular dystrophy. Ann Intern Med 119: 900-905.

Galassi G, Modena MG, Benassi A, Nemni R, Gibertoni M, Volpi G, Colombo A (1986) Autosomal-dominant dystrophy with humeroperoneal weakness and cardiopathy: genetic variant of Emery-Dreifuss disease? Ital J Neurol Sci 7: 125-132.

George J, Patal S, Wexler D, Roth A, Sheps D, Keren G (2005) Circulating matrix metalloproteinase-2 but not matrix metalloproteinase-3, matrix metalloproteinase9 , or tissue inhibitor of metalloproteinase- 1 predicts outcome in patients with congestive heart failure. Am Heart J 150: 484-487.

Goldsmith EC, Borg TK (2002) The dynamic interaction of the extracellular matrix in cardiac remodeling. J Card Fail 8 (Suppl. 6): S314-S318. 
Kähäri VM, Saarialho-Kere U (1999) Matrix metalloproteinases and their inhibitors in tumour growth and invasion. Ann Med 31: 34-45.

Li YY, Feldman AM, Sun Y, McTiernan CF (1998) Differential expression of tissue inhibitors of metalloproteinases in the failing human heart. Circulation 98: 1728-1734.

Li YY, McTiernan CF, Feldman AM (2000) Interplay of matrix metalloproteinases, tissue inhibitors of metalloproteinases and their regulators in cardiac matrix remodeling. Cardiovasc Res 46: 214-224.

MacNaul KL, Chartrain N, Lark M, Tocci MJ, Hutchinson NI (1990) Discordinate expression of stromelysin, collagenase, and tissue inhibitor metalloproteinase- 1 in rheumatoid human synovial fibroblasts: synergistic effects of interleukin- 1 and tumor necrosis factor- $\alpha$ on stromelysin expression. J Biol Chem 265: 17238-17245.

Marriott JB, Goldman JH, Keeling PJ, Baig MK, Dalgleish AG, McKenna WJ (1996) Abnormal cytokines profiles in patients with idiopathic dilated cardiomyopathy and their asymptomatic relatives. Heart 75: 287-290.

Miller RG, Layzer RB, Mellenthin MA, Golabi M, Francoz RA, Mall JC (1985) Emery-Dreifuss muscular dystrophy with autosomal dominant transmission. Neurology 35: 1230-1233.

Munger MA, Johnson M, Amber IJ, Callahan KS, Gilbert EM (1996) Circulating concentrations of proinflammatory cytokines in mild or moderate heart failure secondary to ischemic or idiopathic dilated cardiomyopathy. Am J Cardiol 77: 723-727.

Ohtsuka T, Hamada M, Saeki H, Ogimoto A, Hara Y, Shigematsu Y, Higaki J (2003) Serum levels of matrix metalloproteinases and tumor necrosis factor- $\alpha$ in patients with idiopathic dilated cardiomyopathy and effect of carvedilol on these levels. Am J Cardiol 91: 10241027.

Ohtsuka T, Nishimura K, Kurata A, Kurata A, Ogimoto A, Okayama H, Higaki J (2007) Serum matrix metalloproteinase- 3 as a novel marker for risk stratification of patients with nonischemic dilated cardiomyopathy. J Card Fail 13: 752-758.

Pauschinger M, Knopf D, Petschauer S, Doerner A, Poller W, Schwimmbeck PL, Kühl U, Schultheiss HP (1999) Dilated cardiomyopathy is associated with significant changes in collagen type I/III ratio. Circulation 99: 2750-2756.

Picard F, Brehm M, Fassbach M, Pelzer B, Scheuring S, Küry P, Strauwer BE, Schwartzkopff B (2006) Increased cardiac mRNA expression of matrix metalloproteinase1 (MMP-1) and its inhibitor (TIMP-1) in DCM patients. Clin Res Cardiol 95: 261-269.

Pinelli G, Dominici P, Merlini L Di Pasquale G, Granate C, Bonfiglioli S (1987) Cardiologic evaluation in a family with Emery-Dreifuss muscular dystrophy. G Ital Cardiol 17: 589-593 (in Italian).

Reddy HK, Tjahja IER, Campbell SE, Janicki JS, Hayden MR, Tyagi SC (2004) Expression of matrix metalloproteinase activity in idiopathic dilated cardiomyopathy: a marker of cardiac dilatation. Mol Cell Biochem 264: 183-191.

Ries C, Petrides PE (1995) Cytokine regulation of matrix metalloproteinase activity and regulatory dysfunction in disease. Biol Chem Hoppe Seyler 376: 345-355.

Rouet-Benzineb P, Buhler JM, Dreyfus P, Delcourt A, Dorent R, Perennec J, Crozatier B, Harf A, Lafuma C (1999) Altered balance between matrix gelatinases (MMP-2 and MMP-9) and their tissue inhibitors in human dilated cardiomyopathy: potential role of MMP-9 in myosin-heavy chain degradation. Eur J Heart Fail 1: 337-352.
Rowland LP, Fetel M, Olarte M, Hays A, Singh N, Wanat FE (1979) Emery-Dreifuss muscular dystrophy. Ann Neurol 5: 111-117.

Schwartzkopff B, Fassbach M, Pelzer B, Brehm M, Strauer BE (2002) Elevated serum markers of collagen degradation in patients with mild to moderate dilated cardiomyopathy. Eur J Heart Failure 4: 439-444.

Singer CF, Marbaix E, Kokorine I, Lemoine P, Donnez J, Eeckhout Y, Courtoy PJ (2000) The matrix metalloproteinase-1 (MMP-1) expression in the human endometrium is inversely regulated by interleukin-1 alpha and sex steroids. Ceska Gynekol 65: 211-215.

Spinale FG (2002) Matrix metlloproteinases. Regulation and dysregulation in the failing heart. Cir Res 90: 520-530.

Spinale FG, Coker ML, Krombach SR, Mukherjee R, Hallack H, Houk WV, Clair MJ, Kribbs SB, Johnson LL, Peterson JT, Zile MR (1999) Matrix metalloproteinase. Inhibition during the development of congestive heart failure: effects on left ventricular dimensions and function. Circ Res 85: 364-376.

Spinale FG, Coker ML, Heung LJ, Bopnd BR, Gunasinghe HR, Etoh T, Goldberg AT, Zellner JL, Crumbley AJ (2000) A matrix metalloproteinase induction/activation system exists in the human left ventricular myocardium and is upregulated in heart failure. Circulation 102: 1944-1949.

Thomas ChV, Coker BA, Coker ML, Zellner JL, Handy JR, Crumbley AJ $3^{\text {rd }}$, Spinale FG (1998) Increased matrix metalloproteinase activity and selective upregulation in LV myocardium from patients with end-stage dilated cardiomyopathy. Circulation 97: 1708-1715.

Tziakas DN, Chalikias GK, Papaioakeim M, Hatzinikolau EX, Stakos DA, Tentes IK, Papanas N, Kortsaris A, Maltezos E, Hatseras DI (2005) Comparison of levels of matrix metalloproteinase- 2 and -3 in patients with ischemic cardiomyopathy versus nonischemic cardiomyopathy. Am J Cardiol 96: 1449-1451.

Tyagi SC, Kumar SG, Banks J, Fortson W (1995) Co-expression of tissue inhibitor and matrix metalloproteinases in myocardium. I Mol Cell Cardiol 27: 2177-2189.

Tyagi SC, Campbell SE, Reddy HK, Tjahja E, Voelker DJ (1996) Matrix metalloproteinase activity expression in infarcted, non-infarcted and dilated cardiomyopathic human hearts. Mol Cell Biochem 155: 13-21.

Weber KT, Brilla CG (1991) Pathological hypertrophy and cardiac interstitium: fibrosis and rennin-angiotensin-aldosteron system. Circulation 83: 1849-1865.

Woessner JF (1991) Matrix metalloproteinases and their inhibitors in connective tissue remodeling. FASEB J 5: 2145-2154.

van Tintelen JP, Tio RA, Kerstjens-Frederikse S, van Berlo JH, Bopven LG, Suurmeijer A, White SJ, den Dunnenm JT, te Meerman GJ, Vos YJ, van der Hout AH, Osinga J, van den Berg MP, van Veldhuleen DJ, Buys $\mathrm{CH}$, Hofstra RM, Pinto YM (2007) Severe myocardial fibrosis caused by a deletion of the $5^{\prime}$ end of the lamin A/C gene. J Am Coll Card 49: 2430-2439.

Yamazaki T, Lee JD, Shimizu H, Uzui H, Ueda T (2004) Circulating matrix metalloproteinase-2 is elevated in patients with congestive heart failure. Eur J Heart Fail 6: 41-45.

Yoshioka M, Saida K, Itagaki Y, Kamiya T (1989) Follow up study of cardiac involvement in Emery-Dreifuss muscular dystrophy. Arch Dis Child 64: 713-715.

Yokoseki O, Yazaki Y, Suzuki J, Imamura H, Takenaka H, Isobe M (2000) Association of matrix metalloproteinase expression and left ventricle function in idiopathic dilated cardiomyopathy. Jpn Circ J 64: 352-357. 